\title{
WEIGHTS AND DEGREES IN A RANDOM GRAPH MODEL BASED ON 3-INTERACTIONS
}

\author{
ÁGNES BACKHAUSZ, TAMÁS F. MÓRI \\ Department of Probability Theory and Statistics, \\ Eötvös Loránd University \\ Pázmány P. s. 1/C, H-1117 Budapest, Hungary \\ E-mail address: agnes@cs.elte.hu, moritamas@ludens.elte.hu
}

\begin{abstract}
In a random graph model introduced in 1] we give the joint asymptotic distribution of weights and degrees and prove scale-free property for the model. Moreover, we determine the asymptotics of the maximal weight and the maximal degree.
\end{abstract}

\section{INTRODUCTION}

Many random graph models have been invented recently for modelling large networks like the internet or social networks 2, 4. Considering degree distributions, real life networks look quite different from classical (i.e., Erdős-Rényi type) random graphs. Motivated by this observation, in several models [2, 3] the evolution of the graph is driven by the actual degrees. However, in real-world networks larger groups and cliques may also interact and this has a relevant effect on the evolution. Therefore models based on cliques or groups of vertices may be of particular interest [6, 7].

Motivated by that, in [1] we introduced a random graph model with dynamics based on interactions of three vertices. In our model vertices taking part in an interaction together have larger chance to participate together again, thus it is a kind of preferential attachment structure, while in the models referred to earlier there is no possibility to keep track of the number of steps where the members of a given group get new edges together.

In our model vertices, edges and triangles have nonnegative weights, increasing randomly in discrete time steps. The weight is the number of interactions that the vertex, pair of vertices or triplets have participated in. In [1] we investigated the limit of the ratio of vertices of a given weight and proved that they almost surely exist and decay polynomially. This is the scale-free property of the model. We also determined the asymptotics of the weight of a given vertex.

This time we will deal with degrees. This is the number of vertices having interacted with a given one. This is different from the weight of the vertex, which is the total number of interactions. We determine the joint asymptotic distribution of weights and degrees;

Date: 4 June 2012

2010 Mathematics Subject Classification. 05C80, 60G42.

Key words and phrases. Martingale, random graph, preferential attachment, scale-free property.

The European Union and the European Social Fund have provided financial support to the project under the grant agreement no. TÁMOP 4.2.1./B-09/KMR-2010-0003. 
prove scale-free property for degrees; finally, we give the asymptotics of the degree of a given vertex, the maximal weight, and the maximal degree.

\section{THE MODEL}

We start with a single triangle. This has initial weight 1 , and all its three edges have weight 1 . Vertices, edges, and triangles will have nonnegative integer-valued weights, which increase according to the random evolution of the graph.

At each step three vertices will interact. There are two possibilities. With probability $p$, independently of the past, a new vertex is added, which then interacts with two already existing vertices. Otherwise three old vertices interact. We will need $0<p \leq 1$.

Assume that in the $n$th step a new vertex is added to the graph. With probability $r$, independently of the past, the choice is done according to the "preferential attachment" rule, that is, an edge is chosen with probability proportional to its weight, then its endpoints are selected. With probability $1-r$ two distinct old vertices are chosen uniformly at random.

Then the new vertex interacts with the two selected vertices. This means that the triangle they form comes to existence with initial weight 1 , and we increase the weights of all three edges of the 3 -interaction by 1 . This is the end of the step where a new vertex is generated.

With probability $1-p$ three of the old vertices will interact. In such a step we have two choices again. With probability $q$ each triangle is selected with probability proportional to its weight. Otherwise, with probability $1-q$, three distinct vertices will be chosen at random, uniformly, i.e., each triplet with the same probability. This choice is also independent of the past.

In each case, having selected the three vertices to interact, we draw the edges of the triangle that are not present yet. Then the weight of the triangle is increased by 1 , as well as the weights of the three sides of the triangle.

Now we define the weights of vertices. The weight of a vertex is the sum of the weights of the triangles that contain it. Note that this is just the half of the sum of weights of edges from it, because whenever a vertex takes part in an interaction, the first sum is increased by 1 , and the latter one is increased by 2 .

Our model is parametrized by the triplet of probabilities $(p, q, r)$.

This construction was introduced in [1, where the following properties were proved.

The ratio of vertices of weight $w$ converges to $x_{w}$ almost surely as $n \rightarrow \infty$, where

$$
x_{1}=\frac{1}{\alpha+\beta+1}, \quad x_{w}=\frac{\alpha(w-1)+\beta}{\alpha w+\beta+1} x_{w-1},
$$

hence we have

$$
x_{w} \sim \frac{\Gamma\left(1+\frac{\beta+1}{\alpha}\right)}{\alpha \Gamma\left(1+\frac{\beta}{\alpha}\right)} w^{-\left(1+\frac{1}{\alpha}\right)}, \quad \text { as } w \rightarrow \infty
$$

[1, Theorem 3.1].

We have also studied the rate of growth of the weight of a fixed vertex.

It is clear that the weights of the vertices of the starting triangle are interchangeable, therefore it is not necessary to deal with all the three. Let them be labelled by $-2,-1$, and 0 . The further vertices get labels 1,2 , etc, in the order they are added to the graph. Let $D[n, j]$ and $W[n, j]$ denote the degree and the weight of vertex $j$ after step $n$, provided 
it exists. Otherwise let these quantities be equal to zero. Obviously, vertex $j \geq 1$ cannot exist before step $j$.

According to Theorem 4.1 of [1], for $j \geq 0$ fixed we have

$$
W[n, j] \sim \zeta_{j} n^{\alpha} \quad \text { almost surely as } n \rightarrow \infty,
$$

where $\zeta_{j}$ is a positive random variable.

In the sequel we will denote by $\mathcal{F}_{n}$ the $\sigma$-field generated by the first $n$ steps, and by $V_{n}$ the number of vertices after the $n$th step. Thus $V_{0}=3$. Furthermore, let $\mathbb{I}(\cdot)$ be defined as 1 if the condition within the brackets holds, otherwise let it be 0 .

\section{Asymptotic Joint Distribution of DEGREe AND Weight}

We denote the number of vertices of weight $w$ and degree $d$ after $n$ steps by $X[n, d, w]$. When a vertex is born, its initial weight is one, and its initial degree is two. When it takes part in an interaction, its weight is increased by one, while its degree may not change (if it is already connected to the other two interacting vertices), or may increase by one or two. Thus $X[n, d, w]>0$ can occur only for pairs of integers $d$, w with $1 \leq w$ and $2 \leq d \leq 2 w$.

The following theorem is about the almost sure convergence of the ratio of vertices of weight $w$ and degree $d$.

Theorem 3.1. Given integers $1 \leq w$ and $2 \leq d \leq 2 w$ we have

$$
\frac{X[n, d, w]}{V_{n}} \rightarrow x_{d, w}
$$

almost surely as $n \rightarrow \infty$, where the limits $x_{d, w}$ are positive numbers satisfying the following recurrence equation.

$$
\begin{aligned}
x_{2,1} & =\frac{1}{\alpha+\beta+1}, \\
x_{d, w} & =\frac{1}{\alpha w+\beta+1}\left[\alpha_{1}(w-1) x_{d, w-1}+\alpha_{2}(w-1) x_{d-1, w-1}+\beta x_{d-2, w-1}\right]
\end{aligned}
$$

for $w \geq 2$, where

$$
\alpha_{1}=(1-p) q, \quad \alpha_{2}=\frac{2 p r}{3}, \quad \alpha=\alpha_{1}+\alpha_{2}, \quad \beta=\frac{1}{p}[2(1-r)+3(1-p)(1-q)] .
$$

Proof. We compute the conditional expectation of $X[n, d, w]$ with respect to the $\sigma$-algebra $\mathcal{F}_{n-1}$. Note that if an old vertex interacts with a new one, its degree must increase. On the other hand, if we choose vertices with probabilities proportional to certain weights, then no new edges are born between old vertices.

Having built the graph in $n$ steps we consider a fixed vertex with degree $d$ and weight $w$. For simplicity we denote by $V=V_{n}$ the number of vertices after $n$ steps. Then $(d, w)$ can increase

- by $(1,1)$ with probability $\operatorname{pr} \frac{2 w}{3(n+1)}$ (new vertex, preferential attachment);

- by $(1,1)$ with probability $p(1-r) \frac{d}{\left(\begin{array}{c}V \\ 2\end{array}\right)}$, and

by $(2,1)$ with probability $p(1-r) \frac{V-d-1}{\left(\begin{array}{c}V \\ 2\end{array}\right)}$ (new vertex, uniform selection); 
- by $(0,1)$ with probability $(1-p) q \frac{w}{n+1}$ (old vertices, preferential attachment);

- by $(0,1)$ with probability $(1-p)(1-q) \frac{\left(\begin{array}{l}d \\ 2\end{array}\right)}{\left(\begin{array}{c}V \\ 3\end{array}\right)}$,

by $(1,1)$ with probability $(1-p)(1-q) \frac{d(V-d-1)}{\left(\begin{array}{c}V \\ 3\end{array}\right)}$, and

by $(2,1)$ with probability $(1-p)(1-q) \frac{\left(\begin{array}{c}V-d-1 \\ 2\end{array}\right)}{\left(\begin{array}{c}V \\ 3\end{array}\right)}$ (old vertices, uniform selection).

Now it is easy to see that the probability that a vertex of weight $w$ takes part in the interaction at step $n$ is the following (see also [1]).

$$
p\left[r \frac{2 w}{3 n}+(1-r) \frac{2}{V_{n-1}}\right]+(1-p)\left[q \frac{w}{n}+(1-q) \frac{3}{V_{n-1}}\right]=\frac{\alpha w}{n}+\frac{\beta p}{V_{n-1}} ;
$$

this is independent of the degree of the vertex.

For $d=2$ and $w=1$ we also have to take the new vertex into account: a new vertex is born with probability $p$, and its degree is surely 2, while its weight is surely 1 .

Summing up, we obtain the conditional expectation of $X[n, d, w]$ in the following form.

$$
\begin{aligned}
& \mathbb{E}\left(X[n, d, w] \mid \mathcal{F}_{n-1}\right)=X[n-1, d, w]\left(1-\frac{\alpha w}{n}-\frac{\beta p}{V_{n-1}}\right) \\
&+X[n-1, d, w-1](1-p)\left[q \frac{w-1}{n}+(1-q) \frac{\left(\begin{array}{c}
d \\
2
\end{array}\right)}{\left(\begin{array}{c}
V_{n-1} \\
3
\end{array}\right)}\right] \\
&+ X[n-1, d-1, w-1] p\left[r \frac{2(w-1)}{3 n}+(1-r) \frac{d}{\left(\begin{array}{c}
V_{n-1} \\
2
\end{array}\right)}\right] \\
&+ X[n-1, d-1, w-1](1-p)(1-q) \frac{d\left(V_{n-1}-1-d\right)}{2\left(\begin{array}{c}
V_{n-1} \\
3
\end{array}\right)} \\
&+ X[n-1, d-2, w-1] \times \\
& \times\left[p(1-r) \frac{V_{n-1}-d-1}{\left(\begin{array}{c}
V_{n-1} \\
2
\end{array}\right)}+(1-p)(1-q) \frac{\left(\begin{array}{c}
V_{n-1}-1-d \\
2
\end{array}\right)}{\left(\begin{array}{c}
V_{n-1} \\
3
\end{array}\right)}\right] \\
&+ p \mathbb{I}(d=2, w=1) .
\end{aligned}
$$

Introduce the normalizing sequence

$$
c[n, w]=\prod_{i=1}^{n-1}\left(1-\frac{\alpha w}{i}-\frac{\beta p}{V_{i-1}}\right)^{-1}, \quad n \geq 1, w \geq 1 .
$$

At each step a new vertex is born with probability $p$ independently of the past. Hence the law of large numbers can be applied to the number of vertices, yielding that

$$
V_{n}=p n+o\left(n^{1 / 2+\varepsilon}\right)
$$


a.s., for all $0<\varepsilon<\frac{1}{2}$. This implies that

$$
\begin{aligned}
\log c[n, w]=\sum_{i=1}^{n-1}-\log (1 & \left.-\frac{\alpha w}{i}-\frac{\beta}{i+o\left(i^{1 / 2+\varepsilon}\right)}\right) \\
= & \sum_{i=1}^{n-1}\left(\frac{\alpha w}{i}+\frac{\beta}{i}+o\left(i^{-3 / 2+\varepsilon}\right)\right)=(\alpha w+\beta) \sum_{i=1}^{n-1} \frac{1}{i}+O(1)
\end{aligned}
$$

a.s., where the error term is convergent as $n \rightarrow \infty$. Therefore

$$
c[n, w] \sim a_{w} n^{\alpha w+\beta}
$$

a.s. as $n \rightarrow \infty$, where $a_{w}$ is a positive random variable.

For $n \geq 1, w \geq 1, d \geq 2$ set $Z[n, d, w]=c[n, w] X[n, d, w]$. Multiplying both sides of (6) by $c[n, w]$ one can see that $\left(Z[n, d, w], \mathcal{F}_{n}\right)$ is a nonnegative submartingale for all fixed integers $w, d$.

Consider the Doob decomposition $Z[n, d, w]=M[n, d, w]+A[n, d, w]$, where $M[n, d, w]$ is a zero mean martingale, and $A[n, d, w]$ is a predictable increasing process.

$$
\begin{aligned}
M[n, d, w] & =\sum_{i=1}^{n}\left(Z[i, d, w]-\mathbb{E}\left(Z[i, d, w] \mid \mathcal{F}_{i-1}\right)\right), \\
A[n, d, w] & =\mathbb{E} Z[1, d, w]+\sum_{i=2}^{n}\left(\mathbb{E}\left(Z[i, d, w] \mid \mathcal{F}_{i-1}\right)-Z[i-1, d, w]\right) .
\end{aligned}
$$

Let us give an upper bound on the conditional variance of the martingale part. Recall that $c[i, w]$ is $\mathcal{F}_{i-1}$-measurable, and since there is only one interaction at each step, the increment of $X$ can not be greater than three. Using (8) we get that

$$
\begin{aligned}
B[n, d, w] & =\sum_{i=1}^{n} \operatorname{Var}\left(Z[i, d, w] \mid \mathcal{F}_{i-1}\right)=\sum_{i=1}^{n} c[i, w]^{2} \operatorname{Var}\left(X[i, d, w] \mid \mathcal{F}_{i-1}\right) \\
& =\sum_{i=1}^{n} c[i, w]^{2} \operatorname{Var}\left(X[i, d, w]-X[i-1, d, w] \mid \mathcal{F}_{i-1}\right) \\
& \leq \sum_{i=1}^{n} c[i, w]^{2} \mathbb{E}\left((X[i, d, w]-X[i-1, d, w])^{2} \mid \mathcal{F}_{i-1}\right) \\
& \leq 9 \sum_{i=1}^{n} c[i, w]^{2}=O\left(n^{2(\alpha w+\beta)+1}\right) .
\end{aligned}
$$

Let us apply Proposition VII-2-4 of Neveu [8] with $f(t)=\sqrt{t} \log t$. We obtain that

$$
M[n, d, w]=o\left(B[n, d, w]^{1 / 2} \log B[n, d, w]\right)=o\left(n^{\alpha w+\beta+1}\right) .
$$

We will later use this estimation to show that $Z[n, d, w] \sim A[n, d, w]$.

Note that $X[n, d, w]=0$ if $2 \leq d \leq 2 w$ does not hold. Hence $x_{d, w}=0$ in all these cases.

We apply induction on $w$. If the weight of a vertex is equal to 1 , then it could not participate in any interactions except the first one, when it was born. Thus its degree must be equal to two. Therefore $X[n, d, 1]$ is zero for $d \neq 2$, and it is the number of vertices of weight 1 for $d=2$. In the case $w=1$ the proposition follows from (1). 
Suppose that the statement holds for all weights less than $w$, and for all possible degrees $2 \leq d \leq 2 w$. Let us compute the asymptotics of $A[n, d, w]$. We start from ([6).

$$
\begin{aligned}
& A[n, d, w]=\mathbb{E} Z[1, d, w] \\
&+\sum_{i=2}^{n} c[i, w] X[i-1, d, w-1](1-p)\left[q \frac{w-1}{i}+(1-q) \frac{\left(\begin{array}{c}
d \\
2
\end{array}\right)}{\left(\begin{array}{c}
V_{i-1} \\
3
\end{array}\right)}\right] \\
&+c[i, w] X[i-1, d-1, w-1] p\left[r \frac{2(w-1)}{3 i}+(1-r) \frac{d}{\left(\begin{array}{c}
V_{i-1} \\
2
\end{array}\right)}\right] \\
&+c[i, w] X[i-1, d-1, w-1](1-p)(1-q) \frac{d\left(V_{i-1}-1-d\right)}{2\left(\begin{array}{c}
V_{i-1} \\
3
\end{array}\right)} \\
&+c[i, w] X[i-1, d-2, w-1] \times \\
& \\
&+\times\left[p(1-r) \frac{V_{i-1}-d-1}{\left(\begin{array}{c}
V_{i-1} \\
2
\end{array}\right)}+(1-p)(1-q) \frac{\left(\begin{array}{c}
V_{i-1}-1-d \\
2
\end{array}\right)}{\left(\begin{array}{c}
V_{i-1} \\
3
\end{array}\right)}\right] \\
&+ c[i, w] p \mathbb{I}(d=2, w=1) .
\end{aligned}
$$

Using the induction hypothesis, the asymptotics of $V_{n}$ in (7) and the regular variation of the normalizing constants $c[n, w]$ with exponent $\alpha w+\beta$ in equation (8), we can compute the asymptotics of $A[n, d, w]$, leaving out all terms that are of smaller order of magnitude than others.

$$
\begin{aligned}
A[n, d, w] \sim \sum_{i=2}^{n} c[i, w] p i x_{d, w-1}(1-p) q \frac{w-1}{i} \\
\quad+c[i, w] p i x_{d-1, w-1} p r \frac{2(w-1)}{3 i} \\
\quad+c[i, w] p i x_{d-2, w-1}\left[2(1-r)+\frac{3(1-p)(1-q)}{p}\right] \\
\sim p \sum_{i=2}^{n} a_{w} i^{\alpha w+\beta}\left[(1-p) q(w-1) x_{d, w-1}+p r \frac{2(w-1)}{3} x_{d-1, w-1}\right. \\
\left.\quad+\left(2(1-r)+\frac{3(1-p)(1-q)}{p}\right) x_{d-2, w-1}\right] \\
\sim p \\
\quad \frac{a_{w} n^{\alpha w+\beta+1}}{\alpha w+\beta+1}\left[(1-p) q(w-1) x_{d, w-1}+p r \frac{2(w-1)}{3} x_{d-1, w-1}\right. \\
\left.\quad+\left(2(1-r)+\frac{3(1-p)(1-q)}{p}\right) x_{d-2, w-1}\right]
\end{aligned}
$$

If $d$ and $w$ satisfy $2 \leq d \leq 2 w$, then there is at least one term on the right-hand side that is positive due to the induction hypothesis. Thus $M[n, d, w]=o(A[n, d, w])$, therefore $Z[n, d, w] \sim A[n, d, w]$ holds almost surely as $n \rightarrow \infty$. Dividing by the normalizing constants $c[n, w]$ we get that $X[n, d, w] \sim x_{d, w} p n$, from which

$$
\frac{X[n, d, w]}{V_{n}} \rightarrow x_{d, w}
$$


a.s. as $n \rightarrow \infty$, where

$$
\begin{aligned}
x_{d, w}= & \frac{1}{\alpha w+\beta+1}\left[(1-p) q(w-1) x_{d, w-1}+p r \frac{2(w-1)}{3} x_{d-1, w-1}\right. \\
& \left.+\left(2(1-r)+\frac{3(1-p)(1-q)}{p}\right) x_{d-2, w-1}\right] \\
= & \frac{1}{\alpha w+\beta+1}\left[\alpha_{1}(w-1) x_{d, w-1}+\alpha_{2}(w-1) x_{d-1, w-1}+\beta x_{d-2, w-1}\right],
\end{aligned}
$$

with

$$
\alpha_{1}=(1-p) q, \quad \alpha_{2}=\frac{2 p r}{3}, \quad \beta=2(1-r)+\frac{3(1-p)(1-q)}{p} .
$$

By this the induction step is completed. Moreover, as we noted before, $x_{d, w}>0$ holds for $2 \leq d \leq 2 w$.

Remark 3.1. The explicit solution of the recurrence equation in Theorem [3.1] can be given in the following form.

For $w \geq 1$ set

$$
c_{w}=(\alpha w+\beta+1)(\alpha(w-1)+\beta+1) \ldots(\alpha+\beta+1) .
$$

Let $S_{n}(0)=1$, and for $1 \leq k \leq n$ define

$$
S_{n}(k)=\sum_{1 \leq i_{1}<i_{2}<\cdots<i_{k} \leq n} i_{1} i_{2} \ldots i_{k} .
$$

Then

$$
x_{d, w}=\frac{1}{c_{w}} \sum_{k=1}^{w} S_{w-1}(w-k)\left(\begin{array}{c}
w-k \\
d-2 k
\end{array}\right) \alpha_{1}^{w-d+k} \alpha_{2}^{d-2 k} \beta^{k-1}, \quad 1 \leq w, 2 \leq d \leq 2 w .
$$

In other words, $x_{d, w}$ is equal to the coefficient of $z^{d-2}$ in the expression

$$
\frac{1}{c_{w}} \prod_{i=1}^{w-1}\left(i\left(\alpha_{1}+\alpha_{2} z\right)+\beta z^{2}\right)
$$

This is not hard to derive, and even easier to check; however, it does not seem to be very convenient for determining the asymptotics of $x_{d, w}$ as $d$ or $w$ tends to infinity. We rather choose another method for it in the next section.

\section{Construction of the two Dimensional Limit Distribution}

Let $W$ be a positive integer valued random variable with distribution $\mathbb{P}(W=w)=$

$x_{w}, w=1,2, \ldots$ In addition, let $\xi_{1} \equiv 2$, and let the random variables $\xi_{2}, \xi_{3}, \ldots$ be independent of each other and of $W$ too; moreover,

$$
\begin{gathered}
\mathbb{P}\left(\xi_{w}=0\right)=\frac{\alpha_{1}(w-1)}{\alpha(w-1)+\beta}, \quad \mathbb{P}\left(\xi_{w}=1\right)=\frac{\alpha_{2}(w-1)}{\alpha(w-1)+\beta}, \\
\mathbb{P}\left(\xi_{w}=2\right)=\frac{\beta}{\alpha(w-1)+\beta} .
\end{gathered}
$$

Define the partial sums $S_{w}=\xi_{1}+\cdots+\xi_{w}$.

Theorem 4.1.

$$
\mathbb{P}\left(S_{W}=d, W=w\right)=x_{d, w}, \quad 1 \leq w, 2 \leq d \leq 2 w
$$


Proof. Clearly, $\mathbb{P}\left(S_{W}=2, W=1\right)=\mathbb{P}(W=1)=x_{1}=x_{2,1}$. In addition we have

$$
\begin{aligned}
\mathbb{P}\left(S_{W}=d, W=w\right)= & \mathbb{P}\left(S_{w}=d, W=w\right) \\
= & \mathbb{P}\left(S_{w}=d\right) \mathbb{P}(W=w) \\
= & \mathbb{P}\left(S_{w-1}=d\right) \mathbb{P}\left(\xi_{w}=0\right)+\mathbb{P}\left(S_{w-1}=d-1\right) \mathbb{P}\left(\xi_{w}=1\right)+ \\
& \left.\quad+\mathbb{P}\left(S_{w-1}=d-2\right) \mathbb{P}\left(\xi_{w}=2\right)\right]\left[\mathbb{P}(W=w-1) \frac{\alpha(w-1)+\beta}{\alpha w+\beta+1}\right] \\
=\mathbb{P} & \left(S_{w-1}=d, W=w-1\right) \frac{\alpha_{1}(w-1)}{\alpha w+\beta+1}+ \\
& \quad+\mathbb{P}\left(S_{w-1}=d-1, W=w-1\right) \frac{\alpha_{2}(w-1)}{\alpha w+\beta+1}+ \\
& +\mathbb{P}\left(S_{w-1}=d-2, W=w-1\right) \frac{\beta}{\alpha w+\beta+1},
\end{aligned}
$$

thus the probabilities $\mathbb{P}\left(S_{W}=d, W=w\right)$ and the limits $\left(x_{d, w}\right)$ satisfy the same recursion.

This, combined with Theorem 3.1 implies that the empirical joint distribution of degree and weight after step $n$ converges almost surely in total variation norm to the distribution of $\left(S_{W}, W\right)$. As a corollary we obtain that the asymptotic weight distribution $\left(x_{w}\right)$ is just the marginal of the joint distribution $\left(x_{d, w}\right)$, that is, $x_{w}=x_{1, w}+\cdots+x_{2 w, w}$.

Theorem 4.2. Suppose both $\alpha_{1}$ and $\alpha_{2}$ are positive. Then

$$
x_{d, w}=x_{w} \cdot \frac{\alpha}{\sqrt{2 \pi \alpha_{1} \alpha_{2} w}}\left(\exp \left(-\frac{\left(\alpha d-\alpha_{2} w\right)^{2}}{2 \alpha_{1} \alpha_{2} w}\right)+O\left(w^{-1 / 2}\right)\right),
$$

as $d$ and hence $w$ tend to infinity; and the term $O$ in the remainder is uniform in $d$.

Proof.

$$
\mathbb{E} \xi_{w}=\frac{\alpha_{2}(w-1)+2 \beta}{\alpha(w-1)+\beta}=\frac{\alpha_{2}}{\alpha}+\frac{\left(\alpha+\alpha_{1}\right) \beta}{\alpha(\alpha(w-1)+\beta)},
$$

hence $\mathbb{E} S_{w}=\frac{\alpha_{2}}{\alpha} w+O(\log w)$. Similarly,

$$
\operatorname{Var}\left(\xi_{w}\right)=\frac{\alpha_{1} \alpha_{2}}{\alpha^{2}}+O\left(\frac{1}{w}\right), \quad \operatorname{Var}\left(S_{w}\right)=\frac{\alpha_{1} \alpha_{2}}{\alpha^{2}} w+O(\log w),
$$

as $w \rightarrow \infty$. The proof can be completed by applying the local limit theorem (Theorem VII.1.5 in [9]) to $S_{w}$. Its conditions are satisfied, namely,

$$
\liminf _{w \rightarrow \infty} \frac{1}{w} \operatorname{Var}\left(S_{w}\right)>0, \quad \limsup _{w \rightarrow \infty} \frac{1}{w} \sum_{j=1}^{w}\left|\xi_{j}-\mathbb{E} \xi_{j}\right|^{3}<\infty .
$$

Hence we have

$$
\sup _{d \in \mathbb{Z}}\left|\sqrt{\operatorname{Var}\left(S_{w}\right)} \mathbb{P}\left(S_{w}=d\right)-\frac{1}{\sqrt{2 \pi}} \exp \left(-\frac{\left(d-\mathbb{E} S_{w}\right)^{2}}{2 \operatorname{Var}\left(S_{w}\right)}\right)\right|=O\left(\frac{1}{\sqrt{w}}\right) .
$$

It easily follows that in (12) $\mathbb{E} S_{w}$ can be replaced with a term differing from it by $O\left(\sqrt{\frac{w}{\log w}}\right)$, and $\operatorname{Var}\left(S_{w}\right)$ with a term differing by $O(\sqrt{w})$. 
From Theorem 4.1 one can derive the asymptotics of the other marginal distribution $u_{d}=\sum_{w \geq d / 2} x_{d, w}$. Clearly, $u_{d}$ is the a.s. limit of the proportion of vertices with degree $d$.

Theorem 4.3.

$$
u_{d} \sim \frac{\Gamma\left(1+\frac{\beta+1}{\alpha}\right)}{\alpha_{2} \Gamma\left(1+\frac{\beta}{\alpha}\right)}\left(\frac{\alpha}{\alpha_{2}} d\right)^{-\left(1+\frac{1}{\alpha}\right)}, \quad \text { as } d \rightarrow \infty .
$$

Proof. Let

$$
\begin{gathered}
f=\frac{\alpha}{\alpha_{2}} d, \quad H=\left\{w: f-f^{1 / 2+\varepsilon} \leq w \leq f+f^{1 / 2+\varepsilon}\right\}, \\
H^{-}=\left\{w: w<f-f^{1 / 2+\varepsilon}\right\}, \quad H^{+}=\left\{w: w>f+f^{1 / 2+\varepsilon}\right\},
\end{gathered}
$$

with some $0<\varepsilon<1 / 6$.

By Hoeffding's well-known exponential inequality (Theorem 2 of [5]) for $w \in H^{-}$we have

$$
\begin{aligned}
\mathbb{P}\left(S_{w} \geq d\right) & \leq \mathbb{P}\left(S_{w}-\mathbb{E} S_{w} \geq d-\frac{\alpha_{2}}{\alpha} w-O(\log w)\right) \\
\leq & \exp \left(-\frac{\left(d-\frac{\alpha_{2}}{\alpha} w-O(\log w)\right)^{2}}{2 w}\right)=\exp \left(-\left(\frac{\alpha_{2}}{\alpha}\right)^{2} \frac{(f-w-O(\log w))^{2}}{2 w}\right) .
\end{aligned}
$$

Here in the numerator $(f-w-O(\log w))^{2} \geq f^{1+2 \varepsilon}-O\left(f^{1 / 2+\varepsilon} \log f\right)$, and in the denominator $w \leq f$. Hence

$$
\mathbb{P}\left(S_{w} \geq d\right) \leq \exp \left(-\frac{\alpha_{2}^{2} f^{2 \varepsilon}}{2 \alpha^{2}}+o(1)\right)
$$

thus we have

$$
\mathbb{P}\left(S_{W}=d, W \in H^{-}\right) \leq(1+o(1)) f \exp \left(-\frac{\alpha_{2}^{2} f^{2 \varepsilon}}{2 \alpha^{2}}\right)=o\left(f^{-\left(1+\frac{1}{\alpha}\right)}\right) .
$$

The case of $w \in H^{+}$can be treated similarly.

$$
\begin{aligned}
\mathbb{P}\left(S_{w} \leq d\right) \leq \mathbb{P}\left(S_{w}-\mathbb{E} S_{w} \leq d\right. & \left.-\frac{\alpha_{2}}{\alpha} w\right) \\
& \leq \exp \left(-\frac{\left(\frac{\alpha_{2}}{\alpha} w-d\right)^{2}}{2 w}\right) \leq \exp \left(-\left(\frac{\alpha_{2}}{\alpha}\right)^{2} \frac{(w-f)^{2}}{2 w}\right) .
\end{aligned}
$$

This time we use the estimate

$$
2(w-f) \geq f^{1 / 2+\varepsilon}+w-f \geq f^{1 / 2+\varepsilon}+(w-f)^{1 / 2+\varepsilon} \geq w^{1 / 2+\varepsilon}
$$

in the numerator, obtaining

$$
\mathbb{P}\left(S_{w} \leq d\right) \leq \exp \left(-\frac{\alpha_{2}^{2} w^{2 \varepsilon}}{8 \alpha^{2}}\right) .
$$

Hence

$$
\mathbb{P}\left(S_{W}=d, W \in H^{+}\right) \leq \sum_{w>f} \exp \left(-\frac{\alpha_{2}^{2} w^{2 \varepsilon}}{8 \alpha^{2}}\right)=o\left(f^{-\left(1+\frac{1}{\alpha}\right)}\right) .
$$


Finally, for $w \in H$

$$
\begin{aligned}
\frac{\left(\alpha d-\alpha_{2} w\right)^{2}}{2 \alpha_{1} \alpha_{2} w}=\frac{\alpha_{2}(f-w)^{2}}{2 \alpha_{1} w}=\frac{\alpha_{2}(f-w)^{2}}{2 \alpha_{1} f}\left(1+O\left(f^{-1 / 2+\varepsilon}\right)\right) & \\
& =\frac{\alpha_{2}(f-w)^{2}}{2 \alpha_{1} f}+O\left(f^{-1 / 2+3 \varepsilon}\right),
\end{aligned}
$$

consequently

$$
x_{d, w} \sim \frac{\Gamma\left(1+\frac{\beta+1}{\alpha}\right)}{\alpha \Gamma\left(1+\frac{\beta}{\alpha}\right)} f^{-\left(1+\frac{1}{\alpha}\right)} \cdot \frac{\alpha}{\sqrt{2 \pi \alpha_{1} \alpha_{2} f}} \exp \left(-\frac{\alpha_{2}(w-f)^{2}}{2 \alpha_{1} f}\right),
$$

as $d \rightarrow \infty$ and $w \in H$. Since

$$
\sum_{w \in H} \frac{\alpha}{\sqrt{2 \pi \alpha_{1} \alpha_{2} f}} \exp \left(-\frac{\alpha_{2}(w-f)^{2}}{2 \alpha_{1} f}\right) \rightarrow \int_{-\infty}^{+\infty} \frac{\alpha}{\sqrt{2 \pi \alpha_{1} \alpha_{2}}} \exp \left(-\frac{\alpha_{2} t^{2}}{2 \alpha_{1}}\right) d t=\frac{\alpha}{\alpha_{2}},
$$

we obtain that

$$
\mathbb{P}\left(S_{W}=d, W \in H\right) \sim \frac{\Gamma\left(1+\frac{\beta+1}{\alpha}\right)}{\alpha_{2} \Gamma\left(1+\frac{\beta}{\alpha}\right)} f^{-\left(1+\frac{1}{\alpha}\right)} .
$$

The proof is completed by (13), (14), and (15) combined.

\section{Maximal Weight, maximal Degree}

In this section our goal is to determine the asymptotics of the maximum of the weights as the number of steps tends to infinity.

Let $I[n, j]$ denote the indicator of the event $\{W[n, j] \geq 1\}$. Moreover, we denote by $J[n, j]$ the indicator of the event that vertex $j$ is born at step $n$, that is, $J[n, j]=$ $I[n, j]-I[n-1, j]$.

We fix $j$, and examine the process $W[n, j]$ as $n$ increases.

In the first lemma we find martingales that we will use later in the proofs. Then we prove that the maximal weight grows at the same pace as the weight of any fixed vertex does, see (3).

Let $j, k, \ell$ be fixed integers, $0 \leq j \leq \ell, 1 \leq k$, and let us introduce the sequences

$$
b[n, k]=\prod_{i=1}^{n}\left(1+\frac{\alpha k}{i}\right)^{-1}, \quad d[n, k, j]=\sum_{i=1}^{n-1} b[i+1, k] \frac{\beta p}{V_{i}}\left(\begin{array}{c}
W[i, j]+k-1 \\
k-1
\end{array}\right),
$$

with $\alpha, \beta$ defined in (4). Note that $b[n, k]$ is deterministic, while $d[n, k, j]$ is random, but $\mathcal{F}_{n-1}$-measurable for all $k$ and $j$. Moreover, we have

$$
b[n, k] \sim b_{k} n^{-k \alpha},
$$

with $b_{k}>0$, as $n \rightarrow \infty$.

Lemma 5.1. Let

$$
Z[n, k, j]=b[n, k]\left(\begin{array}{c}
W[n, j]+k-1 \\
k
\end{array}\right)-d[n, k, j],
$$

then $\left(Z[n, k, j] I[\ell, j], \mathcal{F}_{n}\right), n \geq \ell$, is a martingale. 
Proof. Assuming that vertex $j$ already exists after step $\ell$, the probability that it participates in an interaction at step $n+1 \geq \ell$ is equal to $\frac{\alpha W[n, j]}{n+1}+\frac{\beta p}{V_{n}}$. This implies that, for arbitrary positive integers $k, \ell, n$ we have

$$
\begin{gathered}
\mathbb{E}\left(\left(\begin{array}{c}
W[n+1, j]+k-1 \\
k
\end{array}\right) I[\ell, j] \mid \mathcal{F}_{n}\right)=I[\ell, j]\left(\begin{array}{c}
W[n, j]+k-1 \\
k
\end{array}\right) \\
+I[\ell, j]\left[\frac{\alpha W[n, j]}{n}+\frac{\beta p}{V_{n}}\right]\left[\left(\begin{array}{c}
W[n, j]+k \\
k
\end{array}\right)-\left(\begin{array}{c}
W[n, j]+k-1 \\
k
\end{array}\right)\right] \\
=I[\ell, j]\left(\begin{array}{c}
W[n, j]+k-1 \\
k
\end{array}\right)+I[\ell, j]\left[\frac{\alpha W[n, j]}{n}+\frac{\beta p}{V_{n}}\right]\left(\begin{array}{c}
W[n, j]+k-1 \\
k-1
\end{array}\right) \\
=I[\ell, j]\left(\begin{array}{c}
W[n, j]+k-1 \\
k
\end{array}\right)\left(1+\frac{\alpha k}{n}\right)+I[\ell, j] \frac{\beta p}{V_{n}}\left(\begin{array}{c}
W[n, j]+k-1 \\
k-1
\end{array}\right) .
\end{gathered}
$$

Multiplying both sides by $b[n+1, k]$ we get by definition that

$$
\begin{aligned}
& \mathbb{E}\left(b[n+1, k]\left(\begin{array}{c}
W[n+1, j]+k-1 \\
k
\end{array}\right) I[\ell, j] \mid \mathcal{F}_{n}\right) \\
& =I[\ell, j]\left[b[n, k]\left(\begin{array}{c}
W[n, j]+k-1 \\
k
\end{array}\right)+\frac{\beta p}{V_{n}}\left(\begin{array}{c}
W[n, j]+k-1 \\
k-1
\end{array}\right) b[n+1, k]\right] \\
& =I[\ell, j]\left[b[n, k]\left(\begin{array}{c}
W[n, j]+k-1 \\
k
\end{array}\right)-d[n, k, j]+d[n+1, k, j]\right],
\end{aligned}
$$

which completes the proof of the lemma, since $d[n+1, k, j]$ is $\mathcal{F}_{n}$-measurable.

Lemma 5.2. For arbitrary fixed integers $k \geq 0$ and $1 \leq m \leq n$ define

$$
S[m, n, k]=\sum_{j=m}^{n} \mathbb{E}\left[b[n, k]\left(\begin{array}{c}
W[n, j]+k-1 \\
k
\end{array}\right) I[n, j]\right] .
$$

Then

$$
S[m, n, k] \leq C_{k} \sum_{j=m}^{n} j^{-k \alpha}
$$

with a positive constant $C_{k}$ only depending on $k$.

Proof. We prove this by induction on $k$.

For $k=0$ obviously

$$
S[m, n, 0]=b[n, 0] \sum_{j=m}^{n} \mathbb{E}(I[n, j])=\sum_{j=m}^{n} \mathbb{P}(W[n, j] \geq 1) \leq n-m+1 .
$$

Suppose that the statement of the lemma holds for $k-1$. By Lemma 5.1 we know that $Z[n, k, j] I[\ell, j]$ is a martingale, hence its expectation does not depend on $n$. The difference of martingales is also a martingale, thus we have the same with $J[\ell, j]$. Decomposing 
$I[n, j]$ into the sum of terms $J[\ell, j]$ we obtain that

$$
\begin{aligned}
S[m, n, k]=\sum_{j=m}^{n} \mathbb{E}\left(\sum_{\ell=j}^{n} b[n, k]\left(\begin{array}{c}
W[n, j]+k-1 \\
k
\end{array}\right) J[\ell, j]\right) \\
=\sum_{j=m}^{n} \mathbb{E}\left(\sum_{\ell=j}^{n}(Z[n, k, j]+d[n, k, j]) J[\ell, j]\right) \\
=\sum_{j=m}^{n} \mathbb{E}\left(\sum_{\ell=j}^{n}(Z[\ell, k, j]+d[n, k, j]) J[\ell, j]\right) \\
=\mathbb{E}\left(\sum_{j=m}^{n} \sum_{l=j}^{n}(b[\ell, k]+d[n, k, j]-d[\ell, k, j]) J[\ell, j]\right) .
\end{aligned}
$$

Let us split $S[m, n, k]$ into two parts: $S[m, n, k]=S_{1}[m, n, k]+S_{2}[m, n, k]$, where

$$
\begin{aligned}
S_{1}[m, n, k]=\mathbb{E}\left(\sum_{j=m}^{n} \sum_{\ell=j}^{n} b[\ell, k] J[\ell, j]\right) & \leq \sum_{j=m}^{n} b[j, k] \mathbb{E}\left(\sum_{\ell=j}^{n} J[\ell, j]\right) \\
\leq \sum_{j=m}^{n} b[j, k] & =b_{k} \sum_{j=m}^{n} j^{-k \alpha}(1+o(1)) \leq C_{k}^{\prime} \sum_{j=m}^{n} j^{-k \alpha}
\end{aligned}
$$

For the second part we have

$$
\begin{aligned}
& S_{2}[m, n, k]= \mathbb{E}\left(\sum_{j=m}^{n} \sum_{\ell=j}^{n}(d[n, k, j]-d[\ell, k, j]) J[\ell, j]\right) \\
&= \mathbb{E}\left(\sum_{j=m}^{n} \sum_{\ell=j}^{n} \sum_{i=\ell}^{n-1} b[i+1, k] \frac{\beta p}{V_{i}}\left(\begin{array}{c}
W[i, j]+k-1 \\
k-1
\end{array}\right) J[\ell, j]\right) \\
&=\mathbb{E}\left(\sum_{i=m}^{n-1} b[i+1, k] \frac{\beta p}{V_{i}} \sum_{j=m}^{i} \sum_{\ell=j}^{i}\left(\begin{array}{c}
W[i, j]+k-1 \\
k-1
\end{array}\right) J[\ell, j]\right) \\
&=\mathbb{E}\left(\sum_{i=m}^{n-1} \frac{b[i+1, k]}{b[i, k-1]} \frac{\beta p}{V_{i}} \sum_{j=m}^{i} b[i, k-1]\left(\begin{array}{c}
W[i, j]+k-2 \\
k-1
\end{array}\right) \frac{W[i, j]+k-1}{W[i, j]} I[i, j]\right) .
\end{aligned}
$$

Since $\frac{W[i, j]+k-1}{W[i, j]} \leq k$, we get that

$$
S_{2}[m, n, k] \leq k \sum_{i=m}^{n-1} \frac{b[i+1, k]}{b[i, k-1]} \mathbb{E}\left(\frac{\beta p}{V_{i}} \sum_{j=m}^{i} b[i, k-1]\left(\begin{array}{c}
W[i, j]+k-2 \\
k-1
\end{array}\right) I[i, j]\right) .
$$

For the expectation in the right-hand side we give upper bounds on the events $\left\{V_{i}<\right.$ $(p / 2) i\}$ and $\left\{V_{i} \geq(p / 2) i\right\}$ separately. Remember that $\mathbb{I}(\cdot)$ denotes the indicator of the 
event in brackets. From the induction hypothesis we obtain that

$$
\begin{aligned}
\mathbb{E}\left(\frac{\beta p}{V_{i}} \mathbb{I}\left(V_{i} \geq \frac{p i}{2}\right) \sum_{j=m}^{i} b[i, k-1]\left(\begin{array}{c}
W[i, j]+k-2 \\
k-1
\end{array}\right) I[i, j]\right) & \\
& \leq \frac{2 \beta}{i} S[m, i, k-1] \leq 2 \beta C_{k-1} \frac{1}{i} \sum_{j=m}^{i} j^{-(k-1) \alpha} .
\end{aligned}
$$

By the Hoeffding bound $\mathbb{P}\left(V_{i}<(p / 2) i\right) \leq e^{-\varepsilon i}$ with $\varepsilon>0$ only depending on $p$. Using that $V_{i} \geq 3$ and the trivial bound on the weights we get that

$$
\begin{gathered}
\mathbb{E}\left(\frac{\beta p}{V_{i}} \mathbb{I}\left(V_{i}<\frac{p i}{2}\right) \sum_{j=m}^{i} b[i, k-1]\left(\begin{array}{c}
W[i, j]+k-2 \\
k-1
\end{array}\right) I[i, j]\right) \\
\leq \frac{\beta p}{3} \mathbb{P}\left(V_{i}<\frac{p i}{2}\right) \sum_{j=m}^{i} b[i, k-1]\left(\begin{array}{c}
i+k-2 \\
k-1
\end{array}\right) \\
=O\left(e^{-\varepsilon i} i^{-(k-1) \alpha} i^{k-1} i\right)=o\left(\frac{1}{i} \sum_{j=m}^{i} j^{-(k-1) \alpha}\right)
\end{gathered}
$$

uniformly in $m$. Finally,

$$
\frac{b[i+1, k]}{b[i, k-1]}=O\left(i^{-\alpha}\right) .
$$

Putting all these together we obtain that

$$
\begin{aligned}
& S_{2}[m, n, k] \leq C_{k}^{\prime \prime} \sum_{i=m}^{n} i^{-1-\alpha} \sum_{j=m}^{i} j^{-(k-1) \alpha}=C_{k}^{\prime \prime} \sum_{j=m}^{n} j^{-(k-1) \alpha} \sum_{i=j}^{n} i^{-1-\alpha} \\
& \leq C_{k}^{\prime \prime \prime} \sum_{j=m}^{n} j^{-k \alpha}
\end{aligned}
$$

We can complete the proof by combining (17) and (18).

Next we characterize the growth rate of the maximal weight in the graph. Let $\mathcal{W}_{n}=$ $\max \{W[n, j]:-2 \leq j \leq n\}$, the maximal weight after $n$ steps.

Theorem 5.1. $\mathcal{W}_{n} \sim \mu n^{\alpha}$ almost surely as $n \rightarrow \infty$, where $\mu$ is a finite and positive random variable, namely, $\mu=\sup \left\{\zeta_{j}: j \geq-2\right\}$, with $\zeta_{j}$ defined in (3).

Proof. For $1 \leq m \leq n$ define $M[m, n]=\max \{W[n, j]:-2 \leq j<m\}$. By (3) it is obvious that

$$
\lim _{n \rightarrow \infty} n^{-\alpha} M[m, n]=\max \left\{\zeta_{j}:-2 \leq j<m\right\}
$$

with probability 1 . All we have to do is to show that

$$
\lim _{m \rightarrow \infty} \limsup _{n \rightarrow \infty} n^{-\alpha}\left(\mathcal{W}_{n}-M[m, n]\right)=0
$$

From the proof of Lemma 5.1 it follows that the process

$$
b[n, k]\left(\begin{array}{c}
W[n, j]+k-1 \\
k
\end{array}\right) I[\ell, j], \quad n \geq \ell,
$$


is a submartingale, hence, the same holds for

$$
b[n, k]\left(\begin{array}{c}
W[n, j]+k-1 \\
k
\end{array}\right)=b[n, k]\left(\begin{array}{c}
W[n, j]+k-1 \\
k
\end{array}\right) I[n, j], \quad n \geq j .
$$

Being the maximum of an increasing number of submartingales, the process

$$
b[n, k]\left(\begin{array}{c}
\mathcal{W}_{n}-M[m, n]+k-1 \\
k
\end{array}\right), \quad n \geq m,
$$

is also a submartingale. In addition,

$$
\mathbb{E}\left[b[n, k]\left(\begin{array}{c}
\mathcal{W}_{n}-M[m, n]+k-1 \\
k
\end{array}\right)\right] \leq S[m, n, k] \leq C_{k} \sum_{j=m}^{n} j^{-k \alpha}
$$

by Lemma 5.2, Since

$$
\left(\left[b[n, 1]\left(\mathcal{W}_{n}-M[m, n]\right)\right)^{k} \leq k ! \frac{b[n, 1]^{k}}{b[n, k]} b[n, k]\left(\begin{array}{c}
\mathcal{W}_{n}-M[m, n]+k-1 \\
k
\end{array}\right),\right.
$$

the nonnegative submartingale $b[n, 1]\left(\mathcal{W}_{n}-M[m, n]\right)$ is bounded in $L_{k}$ whenever $k \alpha>1$. Thus it is convergent with probability 1 , and also in $L_{k}$, for every $k \geq 1$. Moreover, by (20) and (21) we have

$$
\mathbb{E}\left(\lim _{n \rightarrow \infty} n^{-\alpha}\left(\mathcal{W}_{n}-M[m, n]\right)\right)^{k} \leq k ! \frac{C_{k}}{b_{k}} \sum_{j=m}^{\infty} j^{-k \alpha} .
$$

From this the monotone convergence theorem gives

$$
\mathbb{E}\left(\lim _{m \rightarrow \infty} \lim _{n \rightarrow \infty} n^{-\alpha}\left(\mathcal{W}_{n}-M[m, n]\right)\right)^{k}=0
$$

if $k>1 / \alpha$, proving (19).

We finally present the asymptotics of the maximal degree as the number of steps tends to infinity. First we will study the growth of the degree of a fixed vertex.

Theorem 5.2. For $j=0,1, \ldots$ we have

$$
D[n, j] \sim \frac{\alpha_{2}}{\alpha} \zeta_{j} n^{\alpha}
$$

almost surely, as $n \rightarrow \infty$, where $\zeta_{j}$ is a positive random variable, defined in (3).

Proof. Starting from the specification of the ways the degree and the weight of a fixed vertex can grow, we can write

$$
\begin{aligned}
& \mathbb{E}\left(I[k, j] D[n+1, j] \mid \mathcal{F}_{n}\right)=I[k, j](D[n, j]+p r \frac{2 W[n, j]}{3(n+1)} \\
&\left.+p(1-r) \frac{2 V_{n}-D[n, j]-2}{\left(\begin{array}{c}
V_{n} \\
2
\end{array}\right)}+3(1-p)(1-q) \frac{V_{n}-D[n, j]-1}{\left(\begin{array}{c}
V_{n} \\
2
\end{array}\right)}\right) \\
&=I[k, j]\left(D[n, j]+\alpha_{2} \frac{W[n, j]}{n+1}+R_{n}\right),
\end{aligned}
$$

if $k \leq n$, where $0 \leq R_{n} \leq \frac{2 p \beta}{V_{n}}$. 
Introduce $\xi_{n}=I[k, j](D[n, j]-D[n-1, j])$, then $0 \leq \xi_{n} \leq 2$, hence by Corollary VII-2-6 of [8] and equation (3) we obtain

$$
\sum_{i \leq n} \xi_{i} \sim \sum_{i \leq n} \mathbb{E}\left(\xi_{i} \mid \mathcal{F}_{i-1}\right)=\sum_{i \leq n}\left(\alpha_{2} \frac{W[i-1, j]}{i}+R_{i-1}\right) \sim \frac{\alpha_{2}}{\alpha} \zeta_{j} n^{\alpha}
$$

a.e. on the event $\{W[k, j] \geq 1\}$. Thus $D[n, j] \sim \frac{\alpha_{2}}{\alpha} \zeta_{j} n^{\alpha}$ on that event. Since we know that $\lim _{k \rightarrow \infty} W[k, j]=\infty$, we have

$$
\mathbb{P}\left(\bigcup_{k}\{W[k, j] \geq 1\}\right)=1
$$

completing the proof.

From Theorems 5.1 and 5.2 the asymptotic behaviour of the maximal degree immediately follows.

Theorem 5.3. Let $\mathcal{D}_{n}$ denote the maximal degree in the graph after $n$ steps. Then

$$
\mathcal{D}_{n} \sim \frac{\alpha_{2}}{\alpha} \mu n^{\alpha}
$$

almost surely as $n \rightarrow \infty$, where $\mu$ is the finite and positive random variable defined in Theorem 5.1

Proof. By the trivial bound $D[n, j] \leq 2 W[n, j]$ we obtain

$$
\begin{aligned}
\max \{D[n, j]:-2 \leq j<m\} & \leq \mathcal{D}_{n} \\
& \leq \max \{D[n, j]:-2 \leq j<m\}+\max \{2 W[n, j]: m \leq j \leq n\} .
\end{aligned}
$$

Multiplying by $n^{-\alpha}$ and letting $n \rightarrow \infty$ we get

$$
\begin{aligned}
\frac{\alpha_{2}}{\alpha} \max \left\{\zeta_{j}:-2 \leq j<m\right\} & \leq \liminf _{n \rightarrow \infty} n^{-\alpha} \mathcal{D}_{n} \leq \limsup _{n \rightarrow \infty} n^{-\alpha} \mathcal{D}_{n} \\
\leq & \frac{\alpha_{2}}{\alpha} \max \left\{\zeta_{j}:-2 \leq j<m\right\}+2 \lim _{n \rightarrow \infty} n^{-\alpha}\left(\mathcal{W}_{n}-M[m, n]\right) .
\end{aligned}
$$

By (19) both sides tend to $\mu \alpha_{2} / \alpha$ as $m \rightarrow \infty$.

\section{REFERENCES}

[1] Backhausz, Á., Móri, T. F., A random graph model based on 3-interactions, Annales Univ. Sci. Budapest., Sect. Comp., 36 (2012), 41-52.

[2] Barabási, A-L., Albert, R., Emergence of scaling in random networks, Science, 286 (1999), 509-512.

[3] Cooper, C., Frieze, A., A general model of web graphs, Random Structures Algorithms, 22 (2003), 311-335.

[4] Durrett, R., Random graph dynamics, Cambridge University Press, 2007.

[5] Hoeffding, W., Probability inequalities for sums of bounded random variables, J. Amer. Statist. Assoc., 58 (1963), 13-30.

[6] Móri, T. F., Random multitrees, Studia Sci. Math. Hungar., 47 (2010), 59-80.

[7] Sridharan, A., Yong Gao, Kui Wu, Nastos, J., Statistical behavior of embeddedness and communities of overlapping cliques in online social networks, INFOCOM, 2011 Proceedings IEEE, (2011), 546550 .

[8] Neveu, J., Discrete-parameter martingales, North-Holland, Amsterdam, 1975.

[9] Petrov, V. V., Sums of Independent Random Variables, Akademie-Verlag, Berlin, 1975. 
Ágnes Backhausz

Department of Probability Theory and Statistics, Eötvös Loránd University, Budapest, Hungary

agnes@cs.elte.hu

Tamás F. Móri

Department of Probability Theory and Statistics, Eötvös Loránd University, Budapest, Hungary

moritamas@ludens.elte.hu 